\title{
Construction of the Jacobian matrix for fluorescence diffuse optical tomography using a perturbation Monte Carlo method
}

\author{
Xiaofeng Zhang \\ Center for In Vivo Microscopy, Duke University Medical Center, Durham, NC, 27710
}

\begin{abstract}
Image formation in fluorescence diffuse optical tomography is critically dependent on construction of the Jacobian matrix. For clinical and preclinical applications, because of the highly heterogeneous characteristics of the medium, Monte Carlo methods are frequently adopted to construct the Jacobian. Conventional adjoint Monte Carlo method typically compute the Jacobian by multiplying the photon density fields radiated from the source at the excitation wavelength and from the detector at the emission wavelength. Nonetheless, this approach assumes that the source and the detector in Green's function are reciprocal, which is invalid in general. This assumption is particularly questionable in small animal imaging, where the mean free path length of photons is typically only one order of magnitude smaller than the representative dimension of the medium. We propose a new method that does not rely on the reciprocity of the source and the detector by tracing photon propagation entirely from the source to the detector. This method relies on the perturbation Monte Carlo theory to account for the differences in optical properties of the medium at the excitation and the emission wavelengths. Compared to the adjoint methods, the proposed method is more valid in reflecting the physical process of photon transport in diffusive media and is more efficient in constructing the Jacobian matrix for densely sampled configurations.
\end{abstract}

Keywords: Fluorescence tomography, perturbation Monte Carlo, reconstruction, preclinical imaging

\section{INTRODUCTION}

Fluorescence imaging of small animals has attracted unprecedented attention in the past decade, thanks to the availability of disease-specific preclinical models and rapid development of targeted probes for molecular imaging [1-3]. A major impetus behind these efforts in preclinical optical molecular imaging is the desire to translate the knowledge acquired on the "bench-top" in basic research to the "bed-side" for clinical research via preclinical research.

Conventional fluorescence imaging is only capable of providing two-dimensional images in the visible spectrum on the surface of the samples and thereby severely limits the application of fluorescence imaging in the preclinical domain. Using near infrared fluorophores and applying tomographic reconstruction techniques, e.g., fluorescence diffuse optical tomography (FDOT) in free-space, fluorescently labeled structures deeply embedded in the mice can be reconstructed at millimeter-scale resolution with sub-picomole sensitivity [4]. This imaging technology has been successfully applied to a number of important preclinical models, e.g., [5-8].

Image reconstruction in FDOT requires solving the forward problem, explicitly or implicitly, which models photon migration in diffusive media. In preclinical FDOT, the anatomy of the animal is highly complex and heterogeneous. It requires highly sophisticated methods to model the forward problem accurately [9-13]. Finite element and Monte Carlo are the most frequently used modeling methods because of their flexibility for arbitrarily complex geometry $[14,15]$. The Monte Carlo method is superior in terms of accuracy, but suffers from low computational efficiency. With the rapid advancement of scientific parallel computing technology in recent years, the Monte Carlo method is gaining momentum for high resolution FDOT because of its unparalleled accuracy and broad validity [4, 16, 17].

In conventional implementations of the Monte Carlo method, the Jacobian is typically computed by taking the product of two adjoint matrices, which are the photon density fields of the source and the detector, respectively. This method is best suited for conventional fiber-based configurations, where the numbers of sources and detectors are relatively small, and where the sources and the detectors are interchangeable. The conventional adjoint method becomes highly inefficient when the number of detectors becomes very large, e.g., in densely sampled free-space FDOT, where the number of detectors can potentially grow to the number of pixels of the CCD camera. The inefficiency is because the photon density field of each source and each detector has to be computed and stored in order to perform multiplication at a later

Multimodal Biomedical Imaging VII, edited by Fred S. Azar, Xavier Intes, Proc. of SPIE Vol. 8216, 821600 - (c) 2012 SPIE · CCC code: 1605-7422/12/\$18 - doi: 10.1117/12.906439 
time. For an imaging configuration where $S$ sources and $D$ detectors are required, and where $V$ voxels are used for image reconstruction, the number of Monte Carlo simulations is $S+D$; and the computer storage required to record the intermediate photon density fields is $(S+D) V$. While on the contrary, using our previously proposed method, the number of Monte Carlo simulations is $S$; and no intermediate data is necessary $[4,15]$.

Another limitation of the adjoint method is that it implicitly assumes that the source and the detector are interchangeable, or, optically equivalent, and therefore the Green's function originating from the position $r$ to the detector is equivalent to the computed photon density field originating from the detector to the position $r$. This assumption is valid for fiber-based configurations, where the sources and detectors are made of the same type of fiber optics. In typical free-space configurations for preclinical FDOT, however, the optical characteristics of the light sources (e.g., scanning laser beam or patterned illumination) and the photodetectors (pixels in lens-coupled CCD camera) are highly different.

We have previously developed Monte Carlo software that has been successfully applied in diffuse optical tomography in human brain functional studies, and subsequently implemented an improved parallel version that is well suited for high resolution imaging for the small animals $[4,18]$. However, our previous Monte Carlo method was limited by the fact that it did not account for the difference in the excitation and emission wavelengths in FDOT. In this article, we propose a new method based on the perturbation Monte Carlo theory to address this limitation.

\section{METHODS}

\subsection{Theory}

In the continuous-wave domain, the fluorescent fluence rate $U$ measured by the detector at $r_{d}$ excited by the source at $r_{s}$ is related to the fluoresce yield distribution $\eta$ via the diffusion equation

$$
U\left(r_{s}, r_{d}\right)=\int_{\Omega} \eta(r) w\left(r_{s}, r_{d}, r\right) d r
$$

where the sensitivity function $w$ is given by

$$
w\left(r_{s}, r_{d}, r\right)=\Phi\left(r_{s}, r\right) G\left(r, r_{d}\right)
$$

The spatially varying sensitivity function $w$ is the product of the fluence rate $\Phi$, aka, photon density fields, at the position $r$ radiated from the source at $r_{s}$ and the Green's function from $r$ to the detector at $r_{d}$. In its discretized form

$$
U\left(r_{s}, r_{d}\right)=\sum_{r} \eta(r) w\left(r_{s}, r_{d}, r\right)
$$

where $r_{s}, r_{d}$, and $r$ become the indices of the sources, the detectors and the voxels of the imaging volume, respectively. In the discretized form, the sensitivity function is commonly referred to as the Jacobian matrix, or, simply the Jacobian. It is apparent from Eq. (3) that the Jacobian $w$ describes the weight of the voxels within the imaging volume in the detected fluoresce signal $U$. The sensitivity function at position $r$ is the product of the two photon density fields: one is radiated from the source $r_{s}$ and measured at $r$; and the other is radiated from the unit source at position $r$ and measured at the detector $r_{d}$. Therefore, the sensitivity function at position $r$ is the weighted photon observed at $r_{d}$, which are radiated from $r_{s}$ and happen to pass $r$.

Using Monte Carlo simulations, one can track the exact pathway of every photon that is generated at a given source and arrives at the specified detector. For all of the voxels along this pathway, the Jacobian is related to the Monte Carlo simulation via 


$$
w=\sum_{n} \exp \left(\sum_{m}-\mu_{a m} l_{n m}\right)
$$

where the subscripts $m$ and $n$ are the indices of tissue types and photons migrating from the source and eventually arriving at the detector, and $l_{n m}$ is the total pathway length of the $n$-th photon in tissue type $m$.

In our previously developed method [4], it was assumed that the Stokes shift of the fluorophore was sufficiently small that the difference in the excitation and emission wavelengths was insignificant, which is generally acceptable for organic fluorophores. In order to improve the accuracy in the forward model for high resolution reconstruction, we propose an improved method that account for the wavelength difference. Specifically, we applied the theory of perturbation Monte Carlo and used a correction factor in the Jacobian to compensate the error in the Green's function due to wavelength discrepancy.

In the perturbation Monte Carlo theory [19], a conventional Monte Carlo simulation is first performed with the background optical properties, which establishes the baseline photon weight at the detector. When an arbitrary volume of perturbation to the optical properties occurs in the medium, the resulting alteration to the photon weight is predicted by the perturbation in absorption and scattering coefficients

$$
U_{2}=U_{1}\left(\frac{\mu_{s 2} / \mu_{t 2}}{\mu_{s 1} / \mu_{t 1}}\right)^{j}\left(\frac{\mu_{t 2}}{\mu_{t 1}}\right)^{j} \exp \left[-\left(\mu_{t 2}-\mu_{t 1}\right) l\right]
$$

where the subscripts 1 and 2 are baseline and perturbed states, $\mu_{t}=\mu_{a}+\mu_{s}, j$ is the number of scattering events, and $l$ is the length in the perturbed region.

The concept of perturbation theory can be applied to the Jacobian for FDOT by considering the changes in wavelength, and subsequently optical properties, before and after the fluorescence event as a perturbation to the baseline optical properties. Because the change in scattering coefficient alters the distribution of the photon pathway, we constrain the application of the perturbation Monte Carlo theory by assuming that the scattering coefficients remain constant. As the perturbation can happen in any tissue, the correction factor should be applied to each tissue type. Therefore, the Jacobian described in Eq. (4) should be rewritten and the corrected Jacobian should take the form of

$$
w=\sum_{n}\left[\prod_{m} k_{m} \exp \left(-\mu_{a m} l_{n m}\right)\right]
$$

where the tissue-specific correction factor $k_{m}$ is given by

$$
k_{m}=\exp \left[-\left(\mu_{t m 2}-\mu_{t m 1}\right) l_{n m}\right]
$$

\subsection{Simulations and Numeric Studies}

We first validated our implementation of Monte Carlo simulation of photon migration using a well established analytical method for homogeneous slab geometry. We adopted the analytical solution of photon migration through a turbid slab, which applied a partial current boundary condition that accounted for the mismatch of refractive indices at the boundaries [20]. We used our previously developed parallel computing software [4] to simulate photon migration in the turbid medium under the baseline conditions, i.e., at the excitation wavelength. The optical properties of the medium are listed in Table 1. To characterize the proposed perturbation Monte Carlo-based method, we compared its results against those of the analytical method and the conventional Monte Carlo method. In these comparisons, the same slab geometry and source/detector configuration were used. 
Table 1. Optical properties of the slab geometry

\begin{tabular}{|l|l|l|l|l|}
\hline & $\boldsymbol{\mu}_{a}\left(\mathbf{m m}^{-1}\right)$ & $\boldsymbol{\mu}_{\boldsymbol{s}}^{\prime}\left(\mathbf{m m}^{-\mathbf{1}}\right)$ & $\boldsymbol{g}$ & $\boldsymbol{n}$ \\
\hline Excitation wavelength (Set 1) & 0.02 & 1.00 & 0 & 1.37 \\
\hline Emission wavelength (Set 2) & 0.03 & 1.00 & 0 & 1.37 \\
\hline
\end{tabular}

The thickness of the slab medium was set to $20 \mathrm{~mm}$, similar to the typical size of a mouse for preclinical applications. For the analytical method, the slab geometry extended infinitely in the transverse (x-y) plane, whereas for the Monte Carlo method, the slab was confined to $100 \times 100 \mathrm{~mm}$ in the $\mathrm{x}-\mathrm{y}$ plane. The light source was defined as a parallel beam perpendicular to the boundary (along the z-axis, for both methods) and centered at the surface of the slab (for the Monte Carlo method). A linear array of 5 photodetectors was positioned at the other surface, equal-distantly separated by $5 \mathrm{~mm}$. The geometry and the source/detector configuration are illustrated in Fig. 1.

In the Monte Carlo method, the source and the detectors were both circular with a diameter of $1 \mathrm{~mm}$ and a numeric aperture of 1 ; the voxel size was $(0.2 \mathrm{~mm})^{3}$; and the number of photons was 100 millions. The Monte Carlo software recorded the exact pathways of all the photons that were injected from the sources and were eventually collected by the detectors. The sensitivity function at the excitation wavelength (baseline condition), which is the product of the photon density field originated from the source and the Green's function terminated at the detector [Eq. (2)], was computed according to Eq. (4). The correction factors corresponding to different tissue types were computed using Eq. (7) for the emission wavelength and applied to Eq. (6) to correct the Jacobian. The hardware platforms for computing were the same as described previously in [4]: the baseline Monte Carlo simulations were performed on a SGI Altix 4700 shared-

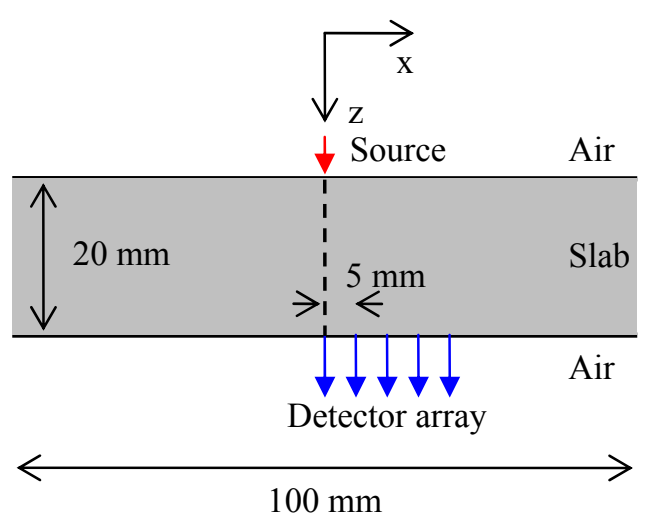

Fig. 1. Geometry and source/detector configuration. memory supercomputer at the Pittsburg Supercomputing Center of Carnegie Mellon University; and data processing and the analytical solutions were computed using a desktop computer workstation.

\section{RESULTS AND DISCUSSION}

A well established analytical solution was used to numerically validate our implementation of Monte Carlo simulation of photon migration in turbid media. Using the geometry shown in Fig. 1 and the optical property Set $1\left(\mu_{a}=0.02, \mu_{s}^{\prime}=1\right.$ $\mathrm{mm}^{-1}, g=0$, and $n=1.37$ ), the fluence rate (intensity of the continuous wave photon density) was evaluated on the surface of the slab where the detectors were positioned (i.e., transmittance measurement) along $\mathrm{z}$-axis at $\mathrm{x}=\mathrm{y}=0$ (the dotted line within the slab between the source and the left-most detector), shown in Fig. 2. The Monte Carlo and the analytical results agree very well with each other. It is noteworthy that in Fig. 2(b), the analytical solution was not plotted for $\mathrm{z}<2 \mathrm{~mm}$ because the diffusion equation is invalid near the source position (comparable to the mean free path length of the tissue), whereas the Monte Carlo result is still valid. 

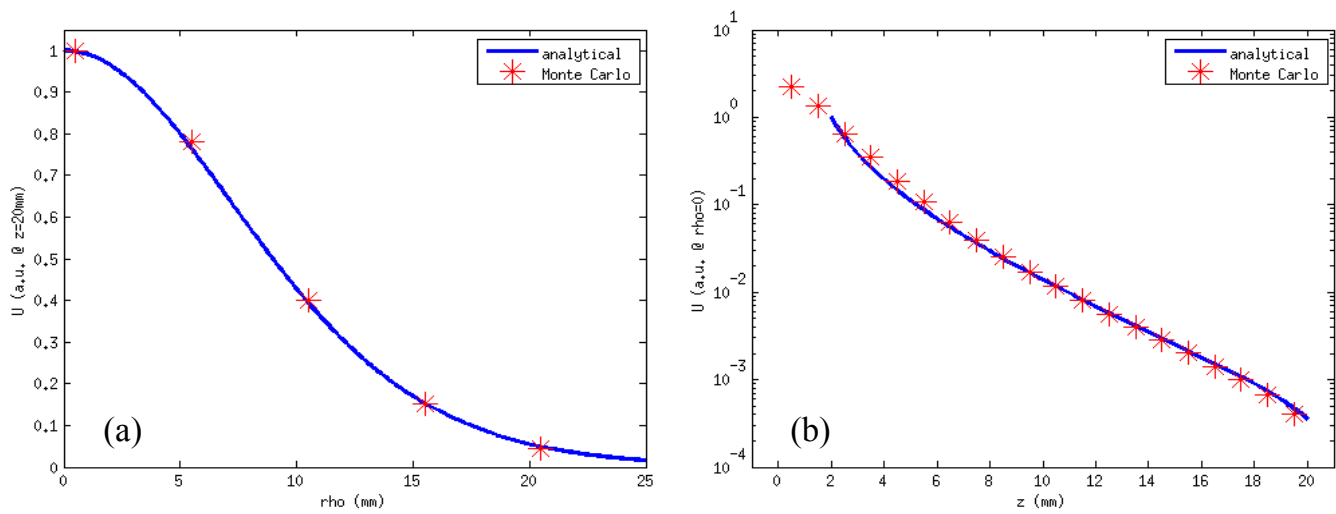

Fig. 2. Comparison of the fluence rate (normalized intensity of the $\mathrm{CW}$ photon density) computed using the Monte Carlo and analytical methods: (a) as a function of the axial distance from the source position at a longitudinal distance of $\mathrm{z}=20 \mathrm{~mm}$; and (b) as a function of the longitudinal distance when the axial distance rho $=0$.

Based on the same baseline Monte Carlo simulation of photon migration, the Jacobian computed using the proposed perturbation method was compared to that using the adjoint method, shown in Fig. 3. Comparing the contour plots of the Jacobian matrices (cross sections along the $z$-axis at $x=y=0$, Figs. 3[a and $b]$ ), It is apparent that the Jacobian computed by the proposed perturbation method (even though the perturbation was nil) was asymmetric and more concentrated toward the source and detector locations; whereas the adjoint method produced a symmetric and smoother result. We theorize that the apparent discrepancy between the two results reflected the modeling limitation of the adjoint method, which implicitly assumes that the source and the detector are optically interchangeable. In addition, it is evident that the Jacobian is noticeably weighted toward the detector side, which will have significant effect on FDOT reconstruction. This effect is more severe in small animal FDOT when the dimension of the animal is not significantly larger than the source-detector distance.
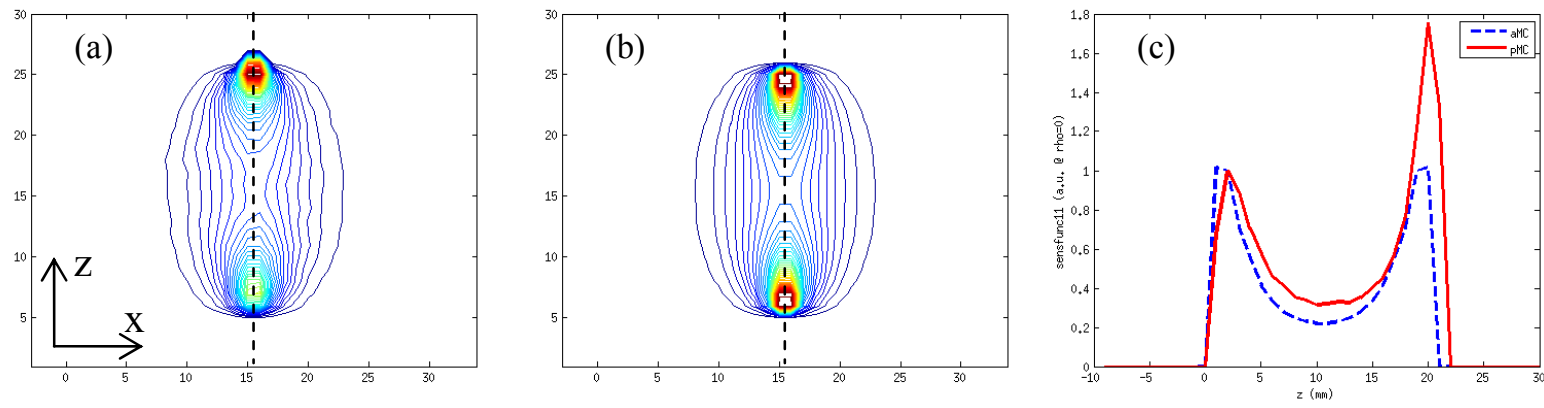

Fig. 3. Comparison of the baseline Jacobian computed using our previously developed and the conventional adjoint methods. ( $a$ and $b$ ) are the contour plots of the Jacobian matrices (cross sections along the $\mathrm{z}$-axis at $\mathrm{x}=\mathrm{y}=0$ ) and $(\mathrm{c})$ is their relative intensity (normalized, perturbation solid and adjoint dotted line) along the $\mathrm{z}$ axis (dotted lines in [a and c]).

Using the optical property Set $2\left(\mu_{a}=0.03, \mu_{s}^{\prime}=1 \mathrm{~mm}^{-1}, g=0\right.$, and $\left.n=1.37\right)$, the perturbation and adjoint Monte Carlo results are compared in the same way as for Set $1\left(\mu_{a}=0.02, \mu_{s}^{\prime}=1 \mathrm{~mm}^{-1}, g=0\right.$, and $\left.n=1.37\right)$. The differences of the Jacobian matrices relative to the baseline condition are presented using contour plots, which showed highly similar patterns, Fig. 4.

It should be pointed out that for the same number of photons (100 millions in this study), the perturbation method produced noisier Jacobians than the adjoint method, shown in Figs. 3(a) and 4(a) vs. Figs. 3(b) and 4(b). Nonetheless, when the number of detectors becomes large, the former is more efficient than the latter in terms of number of simulations $(S$ vs. $S+D)$ and storage space for the intermediate data [nil vs. $(S+D) V]$. 

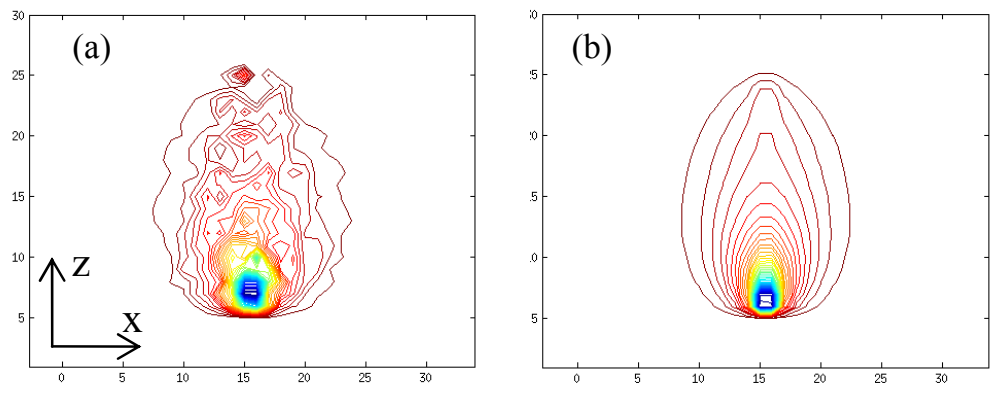

Fig. 4. Comparison of the Jacobians computed using (a) the perturbation and (b) the adjoint methods: the contour plots are the difference of the Jacobians matrices (the same cross sections along the $\mathrm{z}$-axis at $\mathrm{x}=\mathrm{y}=0$ as in Fig. 3).

Another limitation of the proposed method is that it assumes the scattering coefficient remain constant between the excitation and emission wavelengths. This is because in Monte Carlo simulations, the scattering coefficient controls the probability of scattering events (the phase function) and subsequently affects the distribution of photons, irrespective of the absorption coefficient that determines the photon weight.

\section{CONCLUSIONS}

We presented a method to compute the Jacobian matrix for fluorescence diffuse optical tomography, which was based upon the perturbation Monte Carlo theory. Compared to our previously developed method, the proposed perturbation method produced more accurate Jacobian because it accounted for the wavelength difference between the excitation and emission photons. Although the Jacobian matrices produced by the perturbation method were highly similar to those by the conventional adjoint method, the proposed perturbation method is computationally more efficient for densely sampled acquisition, which is necessary for high-resolution FDOT.

\section{ACKNOWLEDGEMENT}

The author is grateful to the funding support by NIH/NCRR R21RR025824 and NCRR P41RR005959, and support of computing by the National Resource for Biomedical Supercomputing (NCRR P41 RR006009) of Pittsburgh Supercomputing Center at Carnegie Mellon University.

\section{REFERENCES}

[1] Grimm, J., Kirsch, D. G., Windsor, S. D., Kim, C. F., Santiago, P. M., Ntziachristos, V., Jacks, T. and Weissleder, R., "Use of gene expression profiling to direct in vivo molecular imaging of lung cancer," Proc. Natl. Acad. Sci. USA 102, 14404-14409 (2005).

[2] Bloch, S., Lesage, F., McIntosh, L., Gandjbakhche, A., Liang, K. and Achilefu, S., "Whole-body fluorescence lifetime imaging of a tumor-targeted near-infrared molecular probe in mice," J. Biomed. Opt. 10, 054003 (2005).

[3] Garofalakis, A., Zacharakis, G., Meyer, H., Economou, E. N., Mamalaki, C., Papamatheakis, J., Kioussis, D., Ntziachristos, V. and Ripoll, J., "Three-dimensional in vivo imaging of green fluorescent protein-expressing T cells in mice with noncontact fluorescence molecular tomography," Mol. Imaging 6, 96-107 (2007).

[4] Zhang, X., Badea, C., Hood, G., Wetzel, A., Qi, Y., Stiles, J. and Johnson, G. A., "High-resolution reconstruction of fluorescent inclusions in mouse thorax using anatomically guided sampling and parallel Monte Carlo computing," Biomed. Opt. Express 2, 2449-2460 (2011).

[5] Niedre, M. J., de Kleine, R. H., Aikawa, E., Kirsch, D. G., Weissleder, R. and Ntziachristos, V., "Early photon tomography allows fluorescence detection of lung carcinomas and disease progression in mice in vivo," Proc. Natl. Acad. Sci. USA 105, 19126-19131 (2008).

[6] McCann, C. M., Waterman, P., Figueiredo, J. L., Aikawa, E., Weissleder, R. and Chen, J. W., "Combined magnetic resonance and fluorescence imaging of the living mouse brain reveals glioma response to chemotherapy," Neuroimage 45, 360-369 (2009).

[7] Hyde, R., de Kleine, R., MacLaurin, S. A., Miller, E., Brooks, D. H., Krucker, T. and Ntziachristos, V., "Hybrid FMT-CT imaging of amyloid-beta plaques in a murine Alzheimer's disease model," Neuroimage 44, 1304-1311 (2009). 
[8] Nahrendorf, M., Waterman, P., Thurber, G., Groves, K., Rajopadhye, M., Panizzi, P., Marinelli, B., Aikawa, E. Pittet, M. J., Swirski, F. K. and Weissleder, R., "Hybrid in vivo FMT-CT imaging of protease activity in atherosclerosis with customized nanosensors," Arterioscler. Thromb. Vasc. Biol. 29, 1444-1451 (2009).

[9] Tan, Y., and Jiang, H., "Diffuse optical tomography guided quantitative fluorescence molecular tomography," Applied Optics 47, 2011-2016 (2008).

[10] Lin, Y., Yan, H., Nalcioglu, O. and Gulsen, G., "Quantitative fluorescence tomography with functional and structural a priori information," Applied Optics 48, 1328-1336 (2009).

[11] Zhang, X., Badea, C. and Johnson, G. A., "Three-dimensional reconstruction in free-space whole-body fluorescence tomography of mice using optically reconstructed surface and atlas anatomy," J. Biomed. Opt. 14, 064010 (2009).

[12] Hyde, D., Schulz, R., Brooks, D., Miller, E. and Ntziachristos, V., "Performance dependence of hybrid x-ray computed tomography/fluorescence molecular tomography on the optical forward problem," J. Opt. Soc. Am. A 26, 919923 (2009).

[13] Davis, S. C., Samkoe, K. S., O'Hara, J. A., Gibbs-Strauss, S. L., Paulsen, K. D. and Pogue, B. W., "Comparing implementations of magnetic-resonance-guided fluorescence molecular tomography for diagnostic classification of brain tumors," J. Biomed. Opt. 15, 051602 (2010).

[14] Arridge, S. R., Schweiger, M., Hiraoka, M. and Delpy, D. T., "A finite element approach for modeling photon transport in tissue," Med. Phys. 20, 299-309 (1993).

[15] Boas, D., Culver, J., Stott, J. and Dunn, A., "Three dimensional Monte Carlo code for photon migration through complex heterogeneous media including the adult human head," Opt. Express 10, 159-170 (2002).

[16] Fang, Q. and Boas, D. A., "Monte Carlo simulation of photon migration in 3D turbid media accelerated by graphics processing units," Opt Express 17, 20178-20190 (2009).

[17] Chen, J., Venugopal, V. and Intes, X., "Monte Carlo based method for fluorescence tomographic imaging with lifetime multiplexing using time gates," Biomed. Opt. Express 2, 871-886 (2011).

[18] Zhang, X., Toronov, V. and Webb, A. G., "Simultaneous integrated diffuse optical tomography and functional magnetic resonance imaging of the human brain," Opt. Express 13, 5513-5521 (2005).

[19] Hayakawa, C. K., Spanier, J., Bevilacqua, F., Dunn, A. K., You, J. S., Tromberg, B. J. and Venugopalan, V., "Perturbation Monte Carlo methods to solve inverse photon migration problems in heterogeneous tissues," Opt. Lett. 26, 1335-1337 (2001).

[20] Contini, D., Martelli, F. and Zaccanti, G., "Photon migration through a turbid slab described by a model based on diffusion approximation. I. Theory," Applied Optics 36, 4587-4599 (1997). 